\title{
Effects of gas pressurization on the interpretation of NMR hydrocarbon measurements in organic rich shales
}

\author{
Son Dang ${ }^{*}$, Carl Sondergeld, and Chandra Rai \\ Mewbourne School of Petroleum and Geological Engineering, University of Oklahoma, USA
}

\begin{abstract}
The estimation of total hydrocarbons (HCs) in place is one of the most important economic challenges in unconventional resource plays. Nuclear magnetic resonance (NMR) has proven to be a valuable tool in directly quantifying both hydrocarbons and brines in the laboratory and the field. Some major applications of NMR interpretation include pore body size distributions, wettability, fluid types, and fluid properties. However, for tight formations, the effects of the factors on NMR relaxation data are intertwined. One purpose of this study is to review the interpretation of NMR response of HCs in a tight rock matrix through illustrated examples.

When comparing NMR data between downhole wireline and laboratory measurement, three important elements need to be considered: 1) temperature differences, 2) system response differences, and 3) pressure (mainly due to the lost gasses.) The effect of temperature on HCs would be presented with experimental results for bulk fluids. Whereas, the effect of pressure is investigated by injecting gas back into rock matrix saturated with original fluids. The experiments were performed within an NMR transparent Daedalus $\mathrm{ZrO}_{2}$ pressure cell, which operates at pressures up to $10,000 \mathrm{psi}$.

The results show that, at ambient temperature and pressure, NMR responds to a fraction of HCs, which is volatile enough to be observed as an NMR relaxation sequence. The invisible fraction of HCs to NMR sequence at ambient condition can be up to $20 \%$ of the total extractable HCs. Molecular relaxation is impacted by fluid viscosity, pore size, and surface affinity. In other words, the fluid with higher viscosity (either due to temperature or gas loss), presenting in smaller pore, or highly affected by the pore surface, will relax faster, and would be partially invisible to NMR, especially in the field. This is critical to the interpretation of NMR response for liquid rich source rocks, in which all of the above molecular relaxing restrictions can be found. Thus, engineers can underestimate movable HCs by using routine core analysis data.
\end{abstract}

\section{Introduction}

Nuclear Magnetic Resonance (NMR) has been proven to be a useful tool to evaluate formation characteristics in both the laboratory and the field. NMR responses are induced during the relaxation of nuclear spins, for a particular pulse sequence. At a specific magnetic-field strength, scanning frequency is tuned to resonate with the Lamour's frequency of hydrogen found in reservoir fluids, such as brine, oils, gases, and bitumen (Brown 1961[1]; Bryan et al., 2002[2]; Hirasaki et al., 2003[3]). Common NMR parameters used to interpret formation properties, include $T_{1}$ as the longitudinal relaxation time, and $\mathrm{T}_{2}$ as the transverse relaxation time. Combination of $\mathrm{T}_{1}$ and $\mathrm{T}_{2}$ relaxation data can provide important information about formation and fluid properties. For conventional reservoirs, NMR has been used to estimate saturated porosity, and pore size distribution (Keynon et al., 1986[4]), from which permeability can be calculated (Coates et al., 1991[5]; Kenyon et al., 1995[6][7]; Straley et al, 1994[8].)

Recently developed applications of NMR, focusing on unconventional tight rocks, include the partitioning of pore surface affinity (oil-wet versus water-wet in shales) (Odusina et al., 2011[9]; Valori and Nicot, 2019[10]) and the characterization of in situ fluids. However, the interpretation is not necessarily straightforward due to the coexistence of multiples fluids within a complicated pore structure (inorganic pores versus organic pores) (Sinha et al., 2017[11]). Fig. 1 presents an example in which the same HCs yield different NMR responses, due to its containment within different pore types. Fig. 1-a is the $\mathrm{T}_{1}-\mathrm{T}_{2}$ map of a preserved sample with dominant inorganic pores (TOC $<0.5 \mathrm{w} \%$ ); whereas Fig. 1-bis the $\mathrm{T}_{1}-\mathrm{T}_{2}$ map of an adjacent preserved sample $(0.73 \mathrm{ft}$ away) with dominant organic pores $(\mathrm{TOC}=5 \mathrm{w} \%$.)

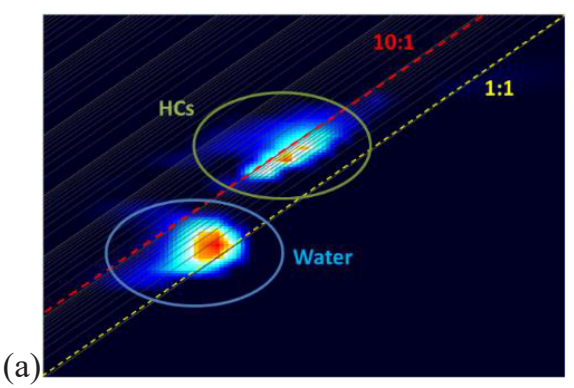

Corresponding author: dangthaison@ou.edu 
(b)

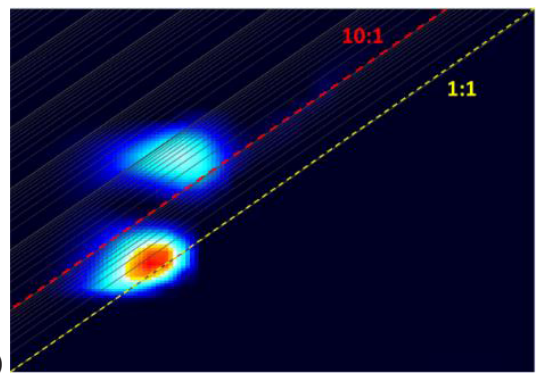

Fig. 1. $T_{1}-T_{2}$ maps of two preserved tight samples with $T_{2}$ is on the $x$-axes, and $T_{1}$ is on the y-axes. Brine NMR response is close to 1:1 line (yellow dash line). Sample (a) with dominant inorganic pores, HCs NMR response is between 1:1 line and 10:1 line (red dash line). Sample (b) with dominant organic pores, HCs NMR response is above 10:1 line, suggesting stronger affinity between HCs and pore surface.

It is essential to verify log-derived parameters using laboratory measurements. Besov et al., 2017[12] demonstrated how the differences in NMR configuration (such as scanning frequency, echo spacing, signal to noise ratio, and magnetic field gradient) can make the comparison between laboratory and field data complicated. Another major concern for almost all laboratory versus log comparison studies is the core condition, even for preserved samples (Blount et al., 2018[13]). In this study, we will review the understanding of NMR response to HCs in bulk fluids as a function of temperature (Dang et al., 2019[14]), as well as the new insights on how gas pressurization/ depressurization influences NMR response on organic rich tight rocks.

\section{Experimental instrument and samples}

NMR distribution, including $T_{2}$ relaxation and $T_{1}-T_{2}$ maps, were acquired at the frequency of $2 \mathrm{MHz}$, using Oxford GeoSpec ${ }^{\mathrm{TM}}$ spectrometers, and Green Imaging acquisition and processing software. The magnet temperature was set at $31^{\circ} \mathrm{C}$ throughout the experiments. The optimal echo spacing, of $114 \mu \mathrm{s}$, was chosen to capture fast relaxation components in the shale samples (including fluids in small pores and heavy HCs components), while preventing the interference of the fluorine signal from internal machine parts.

For pressurization experiments, the samples were placed inside a Daedalus ${ }^{\circledR}$ cell, made of NMR transparent $\mathrm{ZrO}_{2}$; the cell can be operated up to 10,000 psi internal pressure. The cell was positioned inside the NMR spectrometer, in which samples were aligned with the uniform section of the permanent magnetic field. From its inlet, the cell was connected to a Teledyne ISCO syringe pump system, which was used to compress gases from supply cylinders, and then inject gasses into the test cell at a designed pressure. To understand the dynamic response of only the HCs components in rock samples during gas pressurization and depressurization, NMR transparent gasses were chosen. Injected gas candidates include $\mathrm{CO}_{2}, \mathrm{~N}_{2}$, or $\mathrm{CD}_{4}$ (deuterated methane). Fig. 2 illustrates major components of the experimental setup. Note there was no confinement applied on rock samples, gasses were injected all around samples.

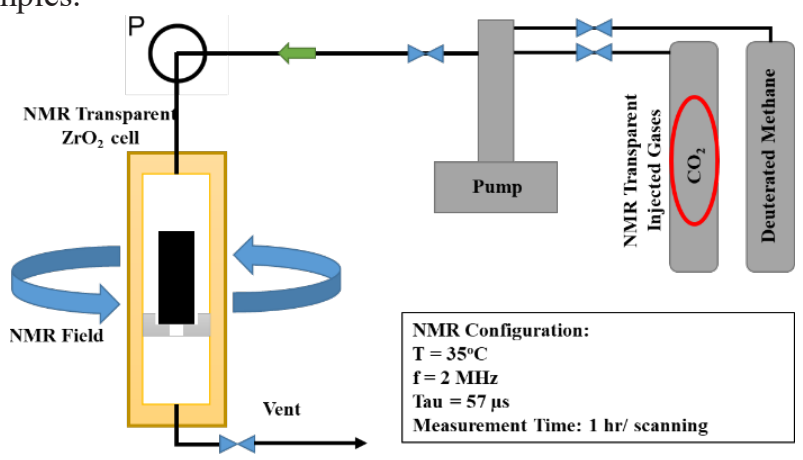

Fig. 2. General experimental setup, including NMR transparent $\mathrm{ZrO}_{2}$ pressure cell, placed within $2 \mathrm{MHz}$ NMR spectrometer. The cell was connected to a pump system to its inlet, and to a vent line from its outlet. NMR transparent injected gasses were chosen, including $\mathrm{CO}_{2}, \mathrm{~N}_{2}$, or deuterated methane.

Four organic rich tight samples were selected for this study from various shale plays; two of them were preserved samples. Routine petrophysical characterizations were performed and presented in Table. 1, including total porosity (the sum of highpressure gas-filled porosity and NMR saturated porosity), mineralogy measured by transmission Fourier Transform Infrared (FTIR), and TOC measured with a LECO $®$ instrument.

\begin{tabular}{|c|c|c|c|c|c|c|c|}
\hline \multirow{2}{*}{$\begin{array}{c}\text { Sample } \\
\text { ID }\end{array}$} & Porosity & TOC & \multicolumn{4}{|c|}{ Mineralogy, w\% } & Sample \\
\cline { 2 - 7 } & p.u & w\% & $\begin{array}{c}\text { Total } \\
\text { Clays }\end{array}$ & $\begin{array}{c}\text { Total } \\
\text { Carbonates }\end{array}$ & $\begin{array}{c}\text { Quartz }+ \\
\text { Feldspars }\end{array}$ & Others & $\begin{array}{c}\text { Non- } \\
\text { preserved }\end{array}$ \\
\hline A & 11.2 & 7.2 & 43 & 0 & 42 & 15 & 1 \\
\hline B & 5.8 & 5 & 33 & 18 & 48 & 1 & Preserved \\
\hline C-1 & 5.1 & 4.9 & 16 & 62 & 13 & 9 & Preserved \\
\hline C-2 & 8.5 & 7.2 & 32 & 45 & 15 & 8 & $\begin{array}{c}\text { Non- } \\
\text { preserved }\end{array}$ \\
\hline
\end{tabular}

Table 1. Petrophysical characterization of four samples. These samples are from three different tight formations.

\section{Measureable fractions of HCs under NMR spectroscopy}

For $\mathrm{T}_{2}$ relaxation acquisition, if a magnetization vector relaxes faster than the setup echo spacing, protons inducing those magnetization vectors cannot be observed with the NMR spectrometer. Fast relaxing components in organic tight rocks include: 1) fluid bound in small pores, 2) highly viscous fluid components (bitumen or heavy alkanes), 3) fluids with strong affinities to pore surfaces and 4) existence of paramagnetic minerals (pyrite or siderite). The existence or coexistence of these factors make the interpretation of NMR response of $\mathrm{HCs}$ in shale samples complicated.

Fig.1 illustrates the impact of surface affinity or pore type on NMR response. With the same HCs, sample (a) with more inorganic pores (or weaker surface affinity), HCs signal was observed in slower relaxation region; whereas, sample (b) with more organic pore type (or stronger surface affinity), HCs signal was observed in a faster relaxation region. 
Dang et al., 2019[14] synthesized a set of six oil samples from the distillation of a same produced crude under inert condition. The crude was heated to elevated temperatures to generate oil samples with successively increasing mean molecular weights. Since NMR measurements were performed at $31^{\circ} \mathrm{C}$, the results suggested that NMR response for these oil samples correlated well with $<$ C17 fraction. Fig. 3 shows good agreement between NMR response and Rock-Eval ${ }^{\circledR} \mathrm{S}_{1}$ intensity (Dang et al., 2019[14]). $\mathrm{S}_{1}$ is associated with the measured fraction of total $\mathrm{HCs}$ with vaporization temperature equal or less than $300^{\circ} \mathrm{C}$.

Previous studies (Chakravarty et al., 2018[15]; Hirasaki et al., 2003[3]) on NMR properties of petroleum reservoir fluids also suggests that besides tool configuration and echo times, NMR response is sensitive to the mobile fraction of HCs, which is clearly a function of fluid composition and temperature.

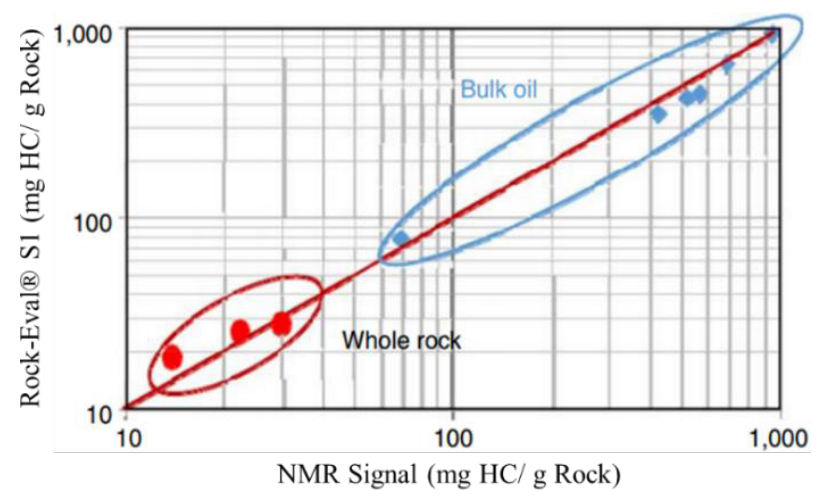

Fig. 3. Comparison between NMR response for HCs and RockEval® $\mathrm{S}_{1}$ (Dang et al., 2019.) $\mathrm{S}_{1}$ intensity represents the fraction of $\mathrm{HCs}$ components vaporized equal to or less than $300^{\circ} \mathrm{C}$, or roughly $<\mathrm{C} 17$ fraction. Blue data points are from bulk oil samples; whereas red data points are from rock samples.

\section{Impact of gas pressurization or depressurization on NMR response}

As previously mentioned, the mobile fraction of HCs is governed by overall fluid composition and temperature. Since NMR response is sensitive to HCs mobility, it is critical to understand the role of gas loss while rock samples are brought up to surface conditions. Gas loss changes overall HCs composition, make the comparison between NMR benchtop measurement and downhole wireline parameters inexact. On the other hands, NMR sensitivity to fluid mobility makes it a useful tool to evaluate $\mathrm{HCs}$ remobilization for gas injection EOR studies.

To focus on the dynamic change of in situ HCs within rock matrix, experiments were performed with NMR transparent gasses. Fig. 4 shows NMR $\mathrm{T}_{2}$ spectra of sample $\mathrm{C}-1$ while $\mathrm{CO}_{2}$ was injected at different pressures. As injection pressure increased, $\mathrm{T}_{2}$ spectra were observed to shift to the slower relaxation region (1$10 \mathrm{~ms}$ ), and signal intensity in the fast relaxation region $(0.1-1 \mathrm{~ms})$ also decreases. These changes suggested viscosity of the original fluid was reduced, or we remobilization of HCs.

Along with the acquisition of $\mathrm{T}_{2}$ relaxation, we also obtained $\mathrm{T}_{1}-\mathrm{T}_{2}$ maps, which help to separate brine from the HCs signals. HCs volume in rock matrix could be calculated by subtracting brine volume from total fluid volume. Fig. 5 shows the normalization of HCs volume in rock matrix as a function of injection pressure. In addition to the shift toward slower $\mathrm{T}_{2}$ relaxation, $\mathrm{HCs}$ volume detected by NMR response also increases, respectively to injection pressure. This result confirmed the existence of a fraction of HCs, which was invisible for NMR under routine laboratory condition. For some samples, the HCs volume can increase by $25 \mathrm{vol} \%$ from initial condition to 5000 psi pressurized condition. Fig. 6 illustrates the remobilization of $\mathrm{HCs}$ during gas injection, which reduces fluid viscosity and bring out a fraction of HCs originally undetectable for NMR.

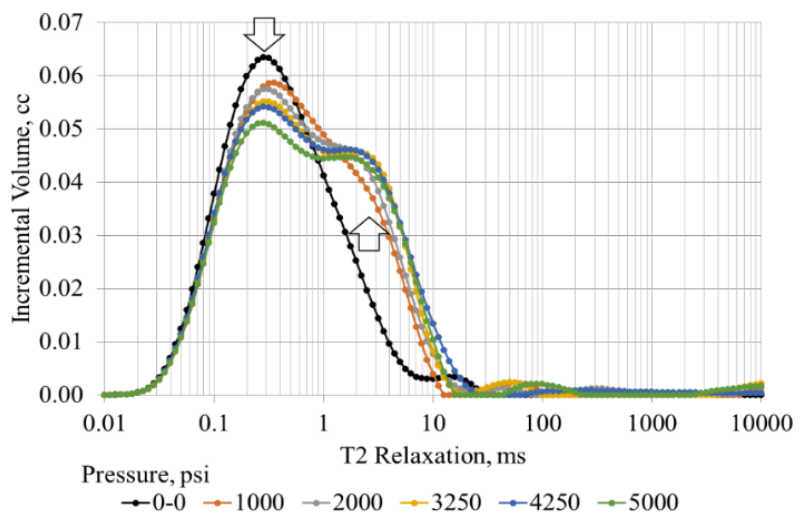

Fig. 4. $\mathrm{T}_{2}$ spectra of sample $\mathrm{C}-1$ with the injection of $\mathrm{CO}_{2}$. Black spectrum is NMR response at original condition. $\mathrm{T}_{2}$ were observed to shift to slower relaxation region $(1-10 \mathrm{~ms})$, and signal intensities in the fast relaxation region $(0.1-1 \mathrm{~ms})$ decreases. These changes suggest the reduction of fluid viscosity.

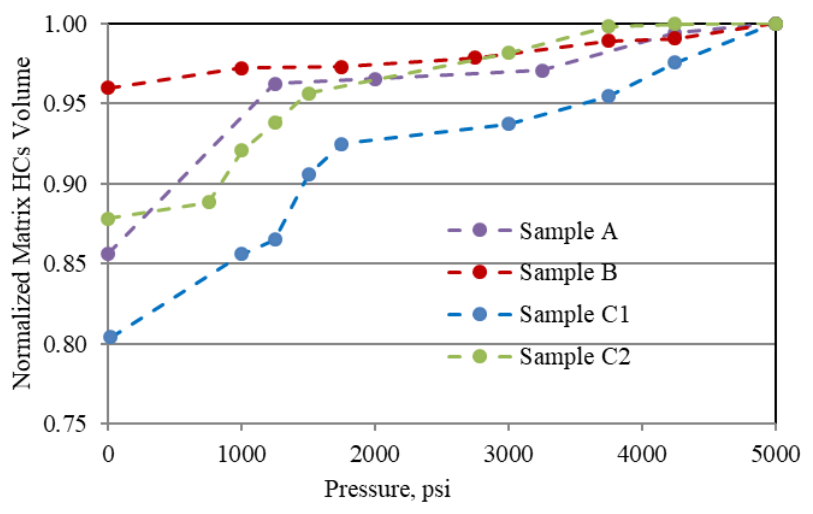

Fig. 5. Normalized HCs volume as a function of injection pressure. HCs volume detected by NMR increases with injection pressure. This suggests the existence of a fraction of $\mathrm{HCs}$, originally invisible for NMR under routine laboratory condition.

Fig. 7 shows the hysteresis of normalized HCs volume as a function of pore pressure between pressurization and depressurization. While pressurization 
data confirms the remobilization of HCs, depressurization data suggests gas trap phenomenon inside the HCs phase during depressurization. Translating to field applications, huff-n-puff EOR can be effective by increasing remaining $\mathrm{HCs}$ ' mobility. Due to gas trapping mechanism, engineers can plan for shorter subsequent injection cycles.

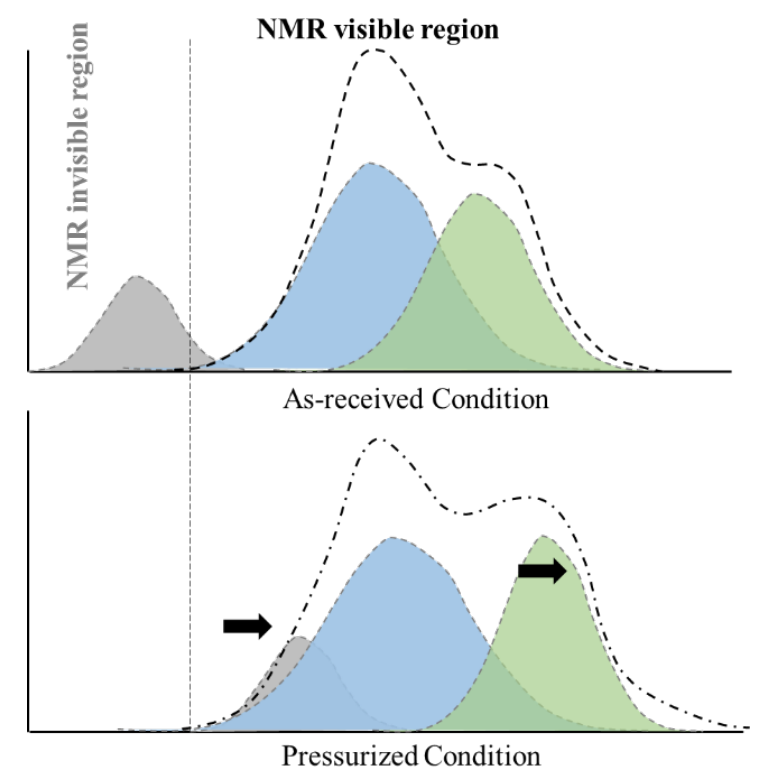

Fig. 6. Scheme for the remobilization of HCs during gas pressurization experiments. Blue peak corresponds to brine, green peak corresponds to light/ originally mobile HCs fraction, and gray peak corresponds to heavy HCs components. Gas pressurization reduces fluid oil viscosity, brings HCs signals toward slower relaxation regions. This makes more HCs detectable by NMR, comparing to the original condition without gas injection.

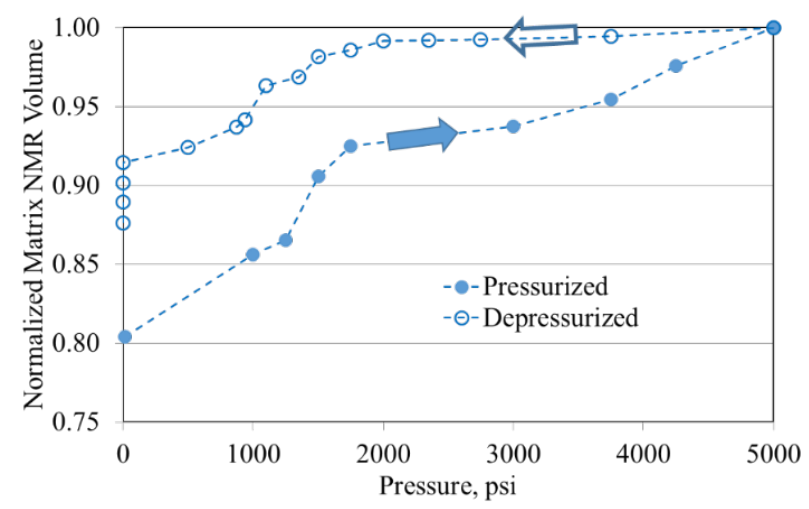

Fig. 7. Normalized HCs volume detectable under NMR as a function of gas pressure during pressurization and depressurization. The hysteresis between two data sets, suggest gas trapping during depressurization.

\section{Application to study huff-n-puff in shales}

The dynamic interaction between injection gas and reservoir fluid is not only a function of pressure, but also a function of injection/production strategy. With the introduction of gas into rock matrix, mobility of reservoir fluid would be enhanced, Fig. 4. Under a certain mass transport mechanism (convective flow or diffusion or both), additional volumes of HCs can be recovered after primary production. Fig. 8 shows $T_{2}$ relaxation response of a shale sample throughout a huffn-puff experiment with $\mathrm{CO}_{2}$ as the injected gas. The experiment included four cycles with subsequent increasing of injection pressure (3750 psi, $4250 \mathrm{psi}$, 5000 psi, 5000 psi). During each cycle, gas injection was conducted for 1 day at a constant pressure, followed by 1-day production phase, in which pressure was reduced to blowdown under a controlled program. $\mathrm{T}_{2}$ spectra were divided into three regions, namely non-movable fluids in matrix, movable fluid in matrix, and expelled fluids, respective to different relaxation regions. During the experiment, the signal of remaining non-movable fluid in matrix did not change; whereas, the signal of movable fluids in matrix reduced, but was volumetric balanced to the increasing of expelled fluid fractions (Fig. 9-a and 9-b).

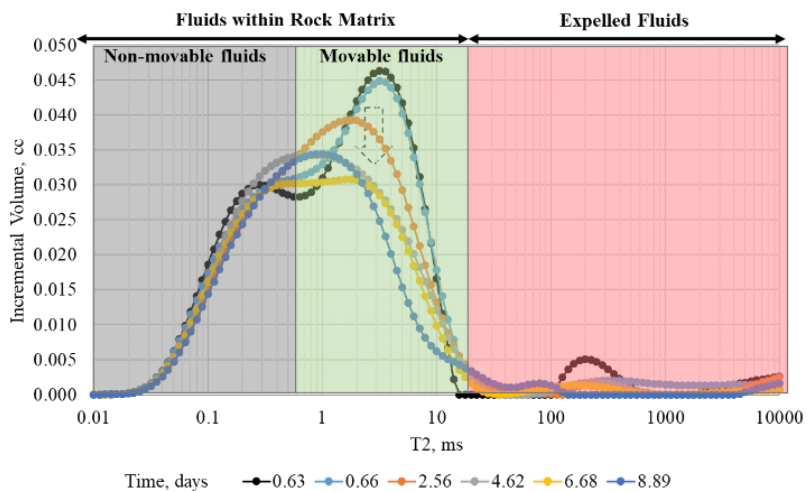

Fig. 8. $T_{2}$ relaxation response of a shale sample throughout a huff-n-puff experiment with $\mathrm{CO}_{2}$ as the injected gas. $\mathrm{T}_{2}$ spectra were divided into three regions, namely non-movable fluids in matrix (gray box, $0.1-1 \mathrm{~ms}$ ), movable fluid in matrix (green box, 1-10ms), and expelled fluids (red box, $>10 \mathrm{~ms}$ ).

(a)

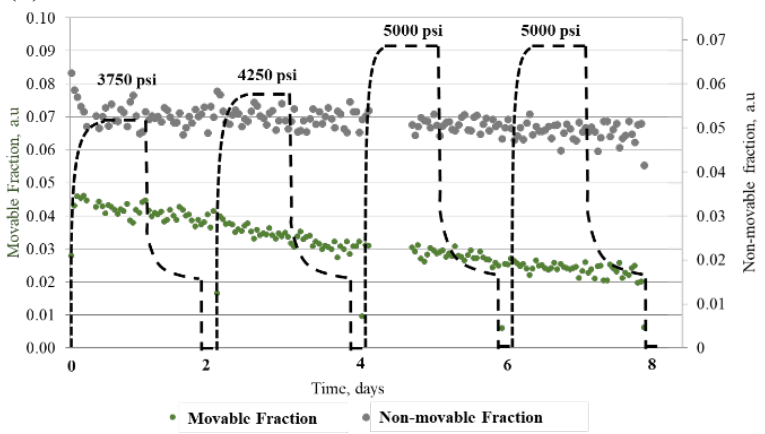

(b)

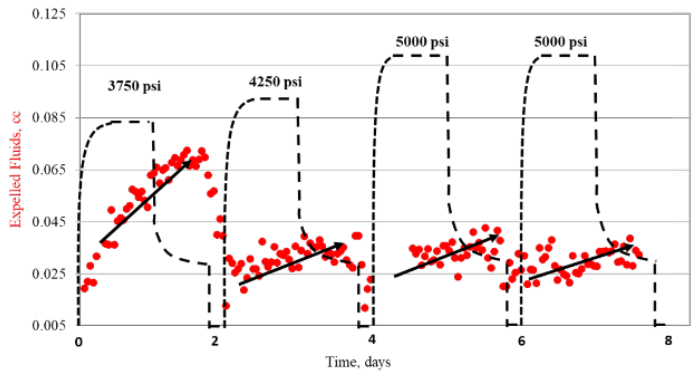


Fig. 9. (a) Concentration profiles of non-movable fluid in matrix (gray) and movable fluid in matrix (green). (b) Concentration profile of expelled fluids (red). While the magnitude of non-movable fluid in matrix did not change during the experiment, movable fluid fraction reduced, but volumetric balanced to expelled/ recovered fluid concentration.

\section{Conclusions}

In this study, we investigate the effect of gas pressurization by injecting gas into a rock matrix saturated with original fluids. The experiments were performed within a NMR transparent $\mathrm{ZrO}_{2}$ pressure cell, placed inside a $2 \mathrm{MHz}$ NMR spectrometer. At a particular temperature and pressure, NMR responds to a fraction of HCs which mobile enough to be observed as an NMR relaxation sequence (roughly $<\mathrm{C} 17$ ). The invisible fraction of HCs to NMR sequence at ambient condition can be as much as $20 \%$ of the total extractable HCs. Molecular relaxation is impacted by fluid viscosity, pore size, and surface affinity. In other words, the HCs fraction with higher viscosity (either due to temperature or gas loss), presenting in smaller pore, or highly affected by the pore surface, will relax faster, and would be partially invisible to NMR. This is critical to the interpretation of NMR response for liquid rich source rocks, in which all above molecular relaxing restrictions can be found. The comparison between downhole wireline parameters and benchtop NMR measures can be incompatible. Engineers can also underestimate movable HCs by using routine core analysis data.

We also demonstrate the use of NMR spectroscopy to evaluate the efficiency of huff-n-puff EOR processes in tight rocks. This technique allows the quantification of $\mathrm{HC}$ recovery without compromising pressure conditions. Relaxation data also provides important knowledge on both fluid remobilization mechanism and mass transportation mechanism, during huff-n-puff.

This work was funded by the University of Oklahoma Unconventional Shale Consortium. We thank the members of Integrated Core Characterization Center $\left(\mathrm{IC}^{3}\right)$ for helping us during experimentation.

\section{References}

1. R. J. S. Brown, "Proton relaxation in oils", Nature 198: 387-388, 1961

2. J. Bryan, F. Manalo, Y. Wen, “Advances in heavy oil and water property measurements using low field nuclear magnetic resonance", SPE International Thermal Operations and Heavy Oil Symposium and International Horizontal Well Technology Conference, Calgary, 4-7 November, 2002, SPE78970-MS

3. G. J. Hirasaki, S. W. Lo, Y. Zhang, "NMR properties of petroleum reservoir fluids", Magnetic Resonance Imaging, Vol. 21, Issues 3-4, p. 269-277, 2003
4. W. W. Kenyon, P. I. Day, C. Straley, J. F. Willemsen, "A three-part study of NMR longitudinal relaxation properties of water-saturated sandstones", SPE Formation evaluation, 622-636, 1986, SPE 15643

5. G. R. Coates, R. C. A. Peveraro, A. Harwick, et al., "The magnetic resonance imaging log characterized by comparison with petrophysical properties and laboratory core data", SPE ATCE, Dallas, 6-9 October, 1991, SPE-22723-MS.

6. B. R. Kenyon, B. R., C. Straley, C., G. Gubelin, G., and C, Morris, "Nuclear magnetic resonance imaging-technology for the $21^{\text {st }}$ century", Oil Field Review, 19-33, 1995

7. W.E. Kenyon, H. H. Takezaki, C. C. Straley, P. N. Sen, P. N., M. Herron, A. Matteson, M. J. Petricola, "A laboratory study of nuclear magnetic resonance relaxation and its relation to depositional texture and petrophysical properties", Carbonate Thamama group, Mubarraz field, Abu Dhai, 1995, SPE 29886.

8. C. D. Straley, C. D. Rossini, H. Vinegar, P. Tutunjian, C. Morriss, "Core analysis by low field NMR”, 1994, SCA-9404

9. E. Odusina, C. H. Sondergeld, C. S. Rai, “An NMR study on shale wettability", Canadian Unconventional Resources Conferences, Calgary, 15-17 November, 2012, SPE-147371-MS.

10. A. Valori, B. Nicot, "A review of 60 years of NMR wettability", Society of Petrophysicists and WellLog Analysts, 2019

11. A. Sinha, S. Dang, C. Sondergeld, C. Rai, "Impact of solvent extraction on surface area measurements in organic-rich shales using nitrogen adsorption", Unconventional Resources Technology Conference, 2017, URTEC-2017-2668849

12. A. Besov, A. Tinni, C. Sondergeld, C. Rai, W. Paul, D. Ebnother, T. Smagala, "Application of laboratory and field NMR to characterize the Tuscaloosa marine shale", Society of Petrophysicists and WellLog Analysts, 2017

13. A. Blount, A. McMullen, M. Durand, T. Croft, B. Driskill, "Maintaining and reconstructing in-situ saturations: a comparison between whole core, sidewall core, and pressurized sidewall core in the Permian basin", Society of Petrophysicists and Well-Log Analysts, 2018

14. S. T. Dang, C. H. Sondergeld, C. S. Rai, "Interpretation of nuclear-magnetic-resonance response to hydrocarbons: application to miscible enhanced-oil-recovery experiments in shales", Society of Petroleum Engineers, 2019

15. A. Chakravarty, A. Tinni, C. S. Rai, C. H. Sondergeld, "NMR considerations in shales at elevated temperature", Unconventional Resources Technology Conference, 2018, URTEC-20182902883 